\title{
LETTER
}

\section{Identification of a potential mechanism of acute kidney injury during the COVID-19 outbreak: a study based on single-cell transcriptome analysis}

\author{
Xiu-wu Pan ${ }^{1,2}$, Da Xü ${ }^{2,5}$, Hao Zhang ${ }^{4,5}$, Wang Zhou ${ }^{2,5^{*}}$, Lin-hui Wang ${ }^{3^{*}}$ and Xin-gang Cui ${ }^{2^{*}}$ (1)
}

(๑) 2020 Springer-Verlag GmbH Germany, part of Springer Nature

\section{Dear Editor,}

Tens of thousands of humans were infected with severe acute respiratory syndrome coronavirus 2 (SARS-CoV-2) within a short period of time, and the infection spread quickly across China and throughout the world. Acute kidney injury (AKI) is one of the important complications of the 2019 novel coronavirus disease (COVID19), occurring in $0.5-7 \%$ of cases and in $2.9-23 \%$ of ICU patients [1-3]. However, whether the AKI of COVID-19 is caused by a coronavirus-induced cytopathic effect or cytokine storm-induced systemic inflammatory response remains unclear.

Similar to SARS-CoV infection, the spike (S) protein of SARS-CoV-2 binds to angiotensin-converting enzyme 2 (ACE2), a host cell receptor, and the $\mathrm{S}$ protein is activated and cleaved by cellular transmembrane serine proteases (TMPRSSs), allowing the virus to release fusion peptides for membrane fusion [4]. Therefore, the coexpression of ACE2 and TMPRSSs is a key determinant for the entry of SARS-CoV-2 into host cells, and improves host conditions for coronavirus.

To deduce the underlying mechanism of AKI during the COVID-19 outbreak, we performed single-cell RNA

\footnotetext{
*Correspondence: brilliant212@163.com; wanglinhui@smmu.edu.cn; cuixingang@smmu.edu.cn

${ }^{2}$ Department of Urology, The Third Affiliated Hospital of Second Military Medical University, 201805 Shanghai, China

${ }^{3}$ Department of Urology, The Changzheng Hospital of Second Military Medical University, 200003 Shanghai, China

Full author information is available at the end of the article

Xiu-wu Pan, Da Xu and Hao Zhang contributed equally to this work, and should be considered as the first author.
}

sequencing (scRNA-seq) analysis to identify candidate kidney host cells in the present study.

First, we drafted the human kidney cell atlas with 42,589 cells from 15 normal kidney samples in three data sets for scRNA-seq analysis (Fig. S1A, Methods in the eSupplement). Subsequently, we clearly identified 19 clusters with significant differences in transcriptional activity and signature gene expression (Fig. S1A-C). Colocalization analysis of ACE2 and TMPRSS genes showed relatively high coexpression in podocytes and proximal straight tubule cells, which were identified as candidate host cells (Fig. 1a, b). The TMPRSS2 gene, as one of the most important mediators of SARS-CoV-2 enter into host cells [4], was found to be coexpressed with ACE2 in podocytes (Fig. S2A).

Second, although there was no significant difference in the expression of TMPRSS genes, the expression of the receptor ACE2 in podocytes and proximal straight tubule cells in Occidental donors was more pronounced than that in Asian donors (Fig. S2B), suggesting that Occidental populations might be at higher risk for developing AKI in COVID-19. In addition, comparative analysis showed that the coexpression of the receptor ACE2 and TMPRSS genes in kidney cells was no less than that in the lung, oesophagus, small intestine and colon (Fig. $\mathrm{S} 2 \mathrm{C}$ ), suggesting that the kidney might also be an important target organ for SARS-CoV-2.

Finally, our study clearly identified podocytes and proximal straight tubule cells as kidney host cells. Podocytes and proximal straight tubule cells play critical roles in urine filtration, reabsorption and excretion. Notably, podocytes are particularly vulnerable to viral and

\section{实



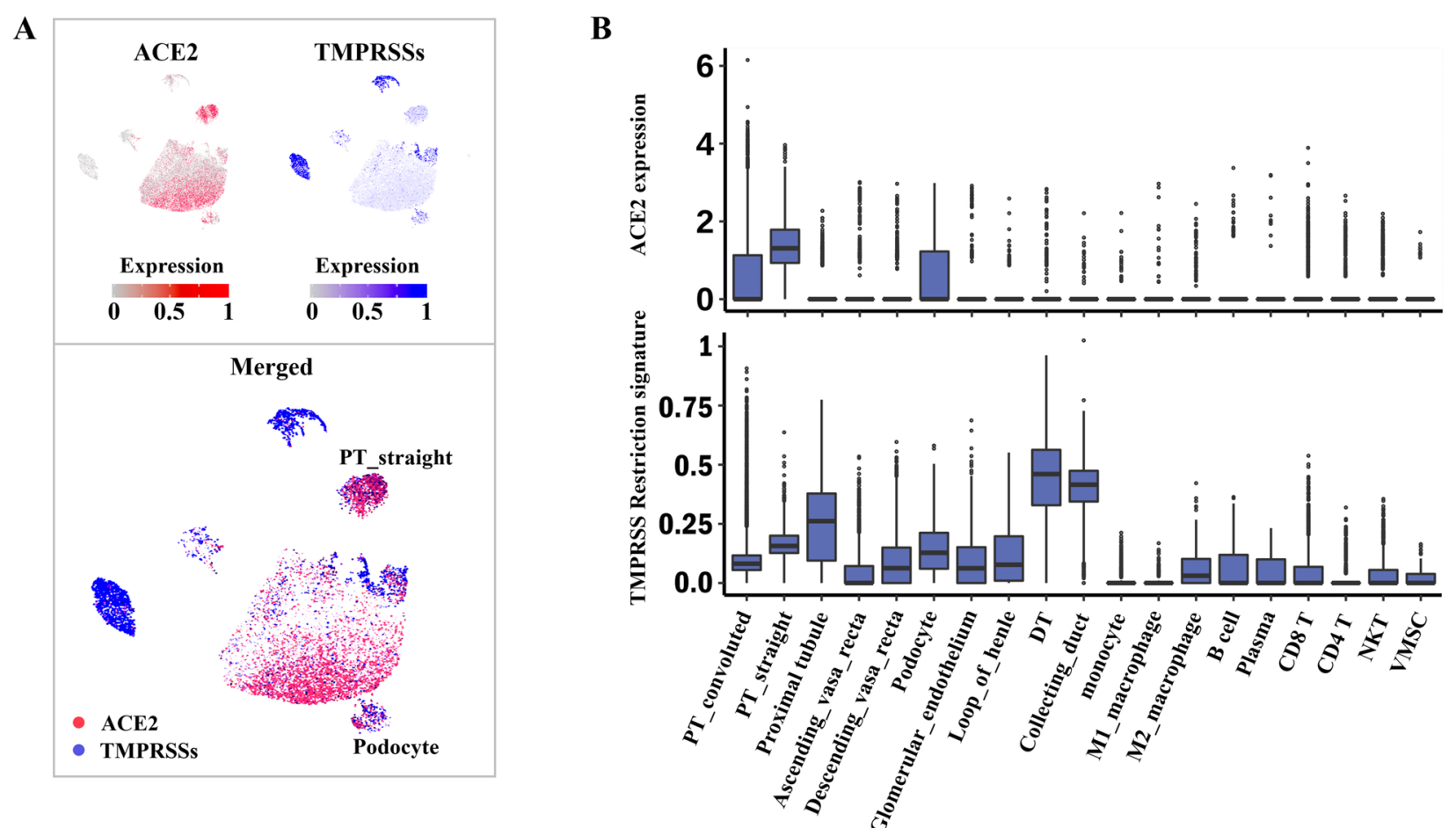

Fig. 1 Identification of kidney host cells by scRNA-seq analysis. a Feature plots show the expression of ACE2 (red) and TMPRSS genes (blue) in kidney epithelial cells. The merged image shows the coexpression of ACE2 and TMPRSS genes, especially in proximal tubules and podocytes. $\mathbf{b}$ Box plots show the expression of ACE2 and the mean expression of the TMPRSS family genes in the 19 clusters of kidney cells. The expression is presented as the $\log 2(\mathrm{TP} 10 \mathrm{~K}+1)$ value

bacterial attacks, and podocyte injury easily induces heavy proteinuria [5]. As recent research data showed, $43.9 \%$ of SARS-CoV-2-infected patients, especially those with AKI, had proteinuria [6]. Moreover, a recent study reported the detection of SARS-CoV-2 infection in urine samples of patients with severe COVID-19 [3]. Furthermore, the entry of SARS-CoV-2 into the systemic circulation is also a key process that leads to AKI. According to published data, the length of time between the detection of SARS-CoV-2 in blood samples and AKI occurrence was approximately 7 days [1].

Based on our findings, we conclude that the cytopathic effects of SARS-CoV-2 on podocytes and proximal straight tubule cells may cause AKI in patients with COVID-19, especially in patients with SARS-CoV-2 infection in blood samples. Therefore, we need to pay more attention to the early monitoring of renal function and cautiously handle the urine of COVID-19 patients with AKI to prevent accidental infection. However, our findings were based on an analysis of normal kidney cells: the proposed mechanism of the pathophysiology of AKI during COVID-19 still needs to be validated in autopsy tissues from COVID-19 patients and functional experiments in animals and cells.
Electronic supplementary material

The online version of this article (https://doi.org/10.1007/s00134-020-06026-1) contains supplementary material, which is available to authorized users.

\begin{abstract}
Author details
1 Department of Urology, The Gongli Hospital of Second Military Medical University, 200135 Shanghai, China. ${ }^{2}$ Department of Urology, The Third Affiliated Hospital of Second Military Medical University, 201805 Shanghai, China. ${ }^{3}$ Department of Urology, The Changzheng Hospital of Second Military Medical University, 200003 Shanghai, China. ${ }^{4}$ Department of Bone Tumor Surgery, The Changzheng Hospital of Second Military Medical University, 200003 Shanghai, China. ${ }^{5}$ Qiu-Jiang Bioinformatics Institute, 200003 Shanghai, China.
\end{abstract}

\section{Acknowledgements}

This work was sponsored by the National Natural Science Foundation of China (No. 81974391); the National Natural Science Foundation of China for Youths (No. 81702515); the Program of Shanghai Academic/Technology Research Leader (No. 19XD1405100); the Shanghai Sailing Program (No. 17YF1425400); The Top-level Clinical Discipline Project of Shanghai Pudong (PWYgf2018-03).

\section{Author contributions}

Xin-gang Cui had full access to all the data in the study and takes responsibility for the integrity of the data and the accuracy of the data analysis. Cui and Zhou contributed to study concept and design. Xu and Zhang contributed to acquisition of data. Pan, Xu and Zhang contributed to analysis and interpretation of data. Pan and Xu contributed to drafting of the manuscript. Cui, Zhou and Wang contributed to critical revision of the manuscript for important intellectual content. Xu and Zhang contributed to statistical analysis. Cui and Pan contributed to obtaining funding. Pan and Xu contributed 
to administrative, technical, or material support. Cui, Wang, Zhou and Pan contributed to supervision.

\section{Compliance with ethical standards}

\section{Conflicts of interest}

All authors declare no conflicts of interest.

\section{Publisher's Note}

Springer Nature remains neutral with regard to jurisdictional claims in published maps and institutional affiliations.

\section{Accepted: 24 March 2020}

Published online: 31 March 2020

\section{References}

1. Huang C, Wang Y, Li X, Ren L, Zhao J, Hu Y, Zhang L, Fan G, Xu J, Gu X, Cheng Z, Yu T, Xia J, Wei Y, Wu W, Xie X, Yin W, Li H, Liu M, Xiao Y, Gao H, Guo L, Xie J, Wang G, Jiang R, Gao Z, Jin Q, Wang J, Cao B (2020) Clinical features of patients infected with 2019 novel coronavirus in Wuhan,
China. Lancet 395(10223):497-506. https://doi.org/10.1016/S0140 $-6736(20) 30183-5$

2. Wang D, Hu B, Hu C, Zhu F, Liu X, Zhang J, Wang B, Xiang H, Cheng Z, Xiong Y, Zhao Y, Li Y, Wang X, Peng Z (2020) Clinical characteristics of 138 hospitalized patients with 2019 novel coronavirus-infected pneumonia in Wuhan, China. JAMA. https://doi.org/10.1001/jama.2020.1585

3. Guan W-J, Ni Z-Y, Hu Y, Liang W-H, Ou C-Q, He J-X, Liu L, Shan H, Lei C-L, Hui DSC, Du B, Li L-J, Zeng G, Yuen K-Y, Chen R-C, Tang C-L, Wang T, Chen P-Y, Xiang J, Li S-Y, Wang J-L, Liang Z-J, Peng Y-X, Wei L, Liu Y, Hu Y-H, Peng P, Wang J-M, Liu J-Y, Chen Z, Li G, Zheng Z-J, Qiu S-Q, Luo J, Ye C-J, Zhu S-Y, Zhong N-S (2020) Clinical characteristics of coronavirus disease 2019 in China. N Engl J Med. https://doi.org/10.1056/NEJMoa2002032

4. Zhang H, Penninger JM, Li Y, Zhong N, Slutsky AS (2020) Angiotensinconverting enzyme 2 (ACE2) as a SARS-CoV-2 receptor: molecular mechanisms and potential therapeutic target. Intensive Care Med. https ://doi.org/10.1007/s00134-020-05985-9

5. Jefferson JA, Nelson PJ, Najafian B, Shankland SJ (2011) Podocyte disorders: core curriculum 2011. Am J Kidney Dis 58(4):666-677. https://doi. org/10.1053/j.ajkd.2011.05.032

6. Cheng Y, Luo R, Wang K, Zhang M, Wang Z, Dong L, Li J, Yao Y, Ge S, Xu $G$ (2020) Kidney disease is associated with in-hospital death of patients with COVID-19. Kidney Int. https://doi.org/10.1016/j.kint.2020.03.005 